\title{
Contents
}

\section{Part I Formalisms}

$1 \quad$ Introductory Information $\ldots \ldots \ldots \ldots \ldots \ldots \ldots \ldots \ldots \ldots$

1.1 Objectives and What You Will Learn from Reading This Book 3

1.2 On Units . . .......................... 4

1.3 Obtaining RSPt and the RSPt Web Site............. 4

1.4 A Short Comment on the History of Linear Muffin-Tin Orbitals and RSPt........................ 4

2 Density Functional Theory and the Kohn-Sham Equation . 7

2.1 The Many-Particle Problem ..................... 8

2.2 Early Attempts to Solve the Many-Particle Problem ........ 10

$2.2 .1 \quad$ Free Electron Model ...................... 10

2.2.2 The Hartree and Hartree-Fock Approaches .......... 10

2.2.3 Thomas-Fermi Theory . . . . . . . . . . . . . . . 11

2.3 Density Functional Theory $\ldots \ldots \ldots \ldots \ldots \ldots \ldots \ldots \ldots \ldots$

2.3.1 Hohenberg-Kohn Theory . .................... 12

2.3.2 The Kohn-Sham Equation .................... 14

2.3 .3 Approximations to $E_{\mathrm{xc}}[n] \ldots \ldots \ldots \ldots \ldots \ldots \ldots$

3 Consequences of Infinite Crystals and Symmetries ........ 21

4 Introduction to Electronic Structure Theory ............. 25

4.1 Born-Oppenheimer Approximation and One-Electron Theory . 25

4.2 Born-von Karman Boundary Condition and Bloch Waves .... 25

4.3 Energy Bands and the Fermi Level .................. 26

4.4 Different Types of k-Space Integration $\ldots \ldots \ldots \ldots \ldots \ldots \ldots 27$

4.5 Self-Consistent Fields . . . . . . . . . . . . . . . . . . . . 31

4.6 Rayleigh-Ritz Variational Procedure .................. 33 
5 Linear Muffin-Tin Orbital Method in the Atomic Sphere

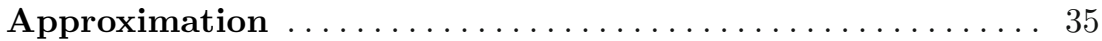

5.1 Muffin-Tin Methods ........................ 35

5.1.1 The Korringa, Kohn, and Rostoker (KKR) Method ... . 36

5.1 .2 The KKR-ASA Method ................... 39

5.1 .3 The LMTO-ASA Method ................ 40

5.1.4 Matrix Elements of the Hamiltonian ............. 42

5.1.5 Logarithmic Derivatives and Choice of the Linearization Energies ...................... 44

5.1.6 Advantages of LMTO-ASA Method.............. 45

6 The Full-Potential Electronic Structure Problem and RSPt 47

6.1 General Aspects ........................... 47

6.1 .1 Notation ............................. 47

6.1.2 Dividing Space: The Muffin-Tin Geometry .......... 49

6.1.3 A Note on the Language of FPLMTO Methods ....... 49

6.2 Symmetric Functions in RSPt ................. 50

6.2.1 The Fourier Grid for Symmetric Functions in RSPt ... 52

6.3 Basis Functions .......................... 52

6.3.1 Muffin-Tin Orbitals...................... 52

6.3.2 FP-LMTO Basis Functions . . . . . . . . . . . . . . 53

6.3.3 Choosing a Basis Set................... 58

6.3.4 Choosing Basis Parameters .................. 58

6.4 Matrix Elements ............................ 62

6.4.1 Muffin-Tin Matrix Elements ................. 62

6.4 .2 Interstitial Matrix Elements . . . . . . . . . . . . . . . . . 63

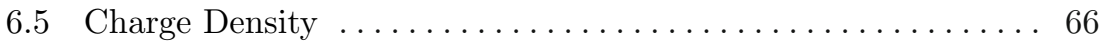

6.6 Core States ............................... 67

6.7 Potential ................................. 67

6.7.1 Coulomb Potential ...................... 67

6.7.2 Density Gradients ...................... 69

6.8 All-Electron Force Calculations................... 69

6.8 .1 Symmetry ........................... 69

6.8.2 Helmann-Feynman and Incomplete Basis Set

Contributions ........................ 70

$7 \quad$ Dynamical Mean Field Theory..................... 75

7.1 Strong Correlations ........................... 75

7.2 LDA/GGA+DMFT Method $\ldots \ldots \ldots \ldots \ldots \ldots \ldots \ldots \ldots \ldots$

7.2 .1 LDA/GGA + U Hamiltonian . . . . . . . . . . . . . 77

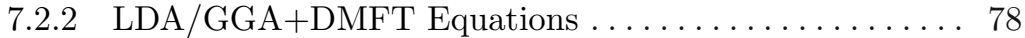

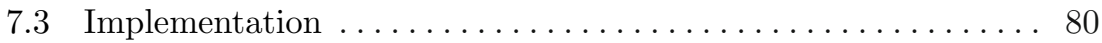

7.3.1 Using the LMTO Basis Set $\ldots \ldots \ldots \ldots \ldots \ldots \ldots . \ldots 1$

7.3 .2 Correlated Orbitals ........................ 82

7.3.3 Other Technical Details ...................... 82 
$7.4 \quad$ Examples.............................. 83

7.4.1 Body-Centered Cubic Iron .................. 83

7.4.2 Systems Close to Localization, the Hubbard-I

Approximation .......................... 85

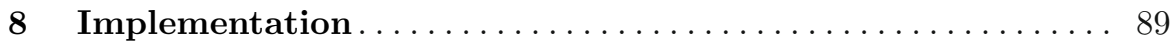

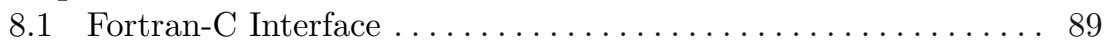

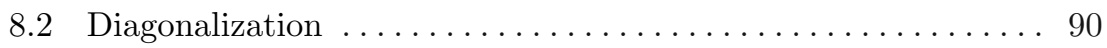

8.3 Fast Fourier Transforms $\ldots \ldots \ldots \ldots \ldots \ldots \ldots \ldots \ldots \ldots \ldots \ldots \ldots$

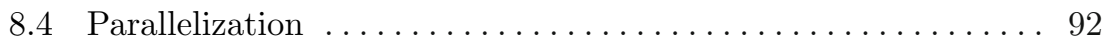

9 Obtaining RSPt from the Web................. 95

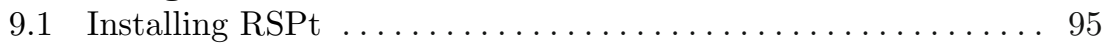

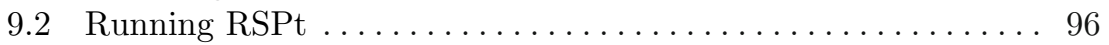

\section{Part II Applications}

10 Total Energy and Forces: Some Numerical Examples ...... 101

10.1 Equation of State............................... 101

10.1.1 Convergence ............................... 105

10.2 Phonon Calculations .......................... 106

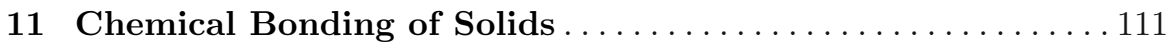

11.1 Electron Densities .............................. 112

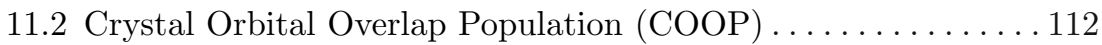

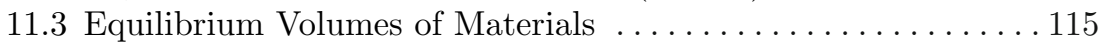

11.3.1 Transition Metals ........................ 116

11.3.2 Lanthanides and Actinides.................... 117

11.3 .3 Compounds . . . . . . . . . . . . . . . . . . . . . 120

11.4 Cohesive Energy . . . . . . . . . . . . . . . . . . . . 121

11.5 Structural Stability and Pressure-Induced Phase Transitions . . 122

11.5.1 An sp-Bonded Material, Ca .................. 122

11.5.2 Transition Metals .......................... 124

11.5.3 Systems with f-Electrons . .................. 125

11.6 Valence Configuration of f-Elements . . . . . . . . . . . . 126

11.7 Elastic Constants . . . . . . . . . . . . . . . . . . . . . 128

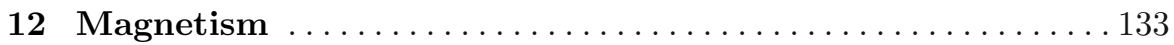

12.1 Spin and Orbital Moments of Itinerant Electron Systems . . . . 134

12.1.1 Symmetry Aspects of Relativistic Spin-Polarized

Calculations .............................136

12.1.2 Elements and Compounds .................. 136

12.1 .3 Surfaces ............................... 138

12.2 Magnetic Anisotropy Energy . . . . . . . . . . . . . . . . . 139 
12.2.1 k-Space Convergence. . . . . . . . . . . . . . . . . . . . 140

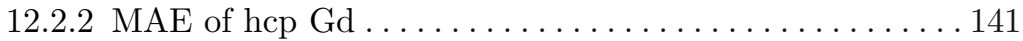

12.3 Magnetism of Nano-objects . . . . . . . . . . . . . . . . 142

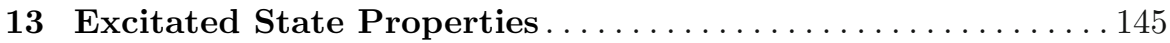

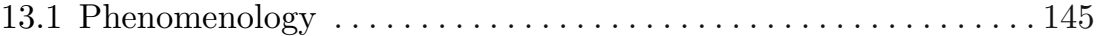

13.1.1 Index of Refraction and Attenuation Coefficient . . . . . 148

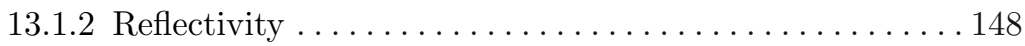

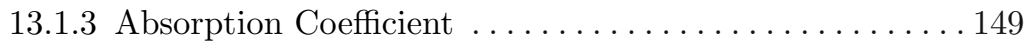

13.1.4 Energy Loss . . . . . . . . . . . . . . . . . . . . 149

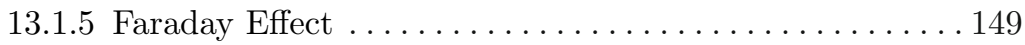

13.1.6 Magneto-optical Kerr Effect . . . . . . . . . . . . 150

13.2 Excited States with DFT: A Contradiction in Terms? . . . . . . . 151

13.3 Quasiparticle Theory versus the Local Density Approximation 152

13.4 Calculation of the Dielectric Function . . . . . . . . . . . . . . 154

13.4.1 Dynamical Dielectric Function . . . . . . . . . . . . . 154

13.4.2 Momentum Matrix Elements . . . . . . . . . . . . 156

13.4.3 Velocity Operator and Sum Rules ............. 158

13.5 Optical Properties of Semiconductors . . . . . . . . . . . . . 159

13.6 Optical Properties of Metals . . . . . . . . . . . . . . . . . 162

13.7 Magneto-optical Properties . . . . . . . . . . . . . . . . . 164

13.8 X-Ray Absorption and X-Ray Magnetic Circular Dichroism . . . 166

13.8.1 The XMCD Formalism . . . . . . . . . . . . . . . 167

13.8.2 The XMCD Sum Rules .................. 170

14 A Database of Electronic Structures . . . . . . . . . . . . 179

14.1 Database Generation. . . . . . . . . . . . . . . . . . . . . 179

14.2 Data-Mining: An Example from Scintillating Materials . . . . . . 180

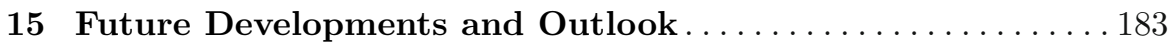

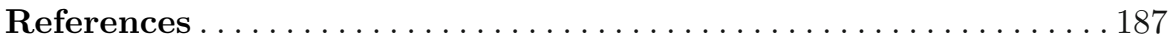

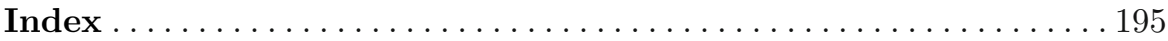

